\title{
The Single Component Thermal Lattice Boltzmann Simulation of Pool Boiling in Two Dimensions*
}

\author{
Takeshi SETA** and Kenichi OKUI** \\ ** Faculty of Engineering, University of Toyama \\ 3190 Gofuku, Toyama-shi, Toyama 930-8555, Japan \\ E-mail: seta@eng.u-toyama.ac.jp
}

\begin{abstract}
In this paper, we propose a lattice Boltzmann model for simulation of two-phase flows pertinent to thermal nonideal fluids in two dimensions. This LBM has a modified pseudo-potential so that it recovers a full set of hydrodynamic equations for two-phase flows through the Chapman-Enskog expansion procedure. Numerical measurements of thermal conductivity and of surface tension agree well with theoretical predictions. Simulations of bubble rising and of pool boiling with heat transfer are carried out. They demonstrate that the model is applicable to two-phase flows with thermal effects. Using finite difference Lattice Boltzmann method ensures numerical stability of the scheme.
\end{abstract}

Key words : Lattice Boltzmann Method, Two-Phase Flows, Computational Fluid Dynamics, Heat Transfer, Pool Boiling, van der Waals-Cahn-Hilliard Free Energy

\section{Introduction}

Due to the highly complicated and hierarchical structures of the two-phase flows, it is difficult to simulate two-phase flows. The interfacial phenomena in the molecular scale control the local and instantaneous phase dynamics in particle scale. The integrated effects of the particle scale phenomena induce transport properties of multi-phase flows in averaged space and time scale. The wide-ranging effects in the averaged space and time scale induce the fluid dynamics that are characteristic of multi-phase flows. The difference of the magnitudes between the films width and the separated phases is ambiguous. To simulate two-phase flows, we have often used an averaging method typical of two-fluid models that use several specific ad hoc assumptions and experimental or empirical constitutive equations. Multi-phase boundary geometries are, however, so complex that the macroscopic approaches using the time and space averaged approximation cannot simulate every bubbles and phase interfaces dynamics.

In recent years, the lattice gas automata $(\mathrm{LGA})^{(1),(2)}$ or the lattice Boltzmann method $(\mathrm{LBM})^{(3),(4)}$ has received considerable attention as an alternative numerical scheme for simulating complex phenomena. In the LGA, the system evolves according to the updating rules that fictitious particles move along lattices with iterating propagation and collision. Accumulation of these particles motion reproduces macroscopic behavior of the overall system. The independent calculation of the individual particles motions brings about not only massively parallel procedure but also easy implementation of complicated boundary geometries. A kinetic equation of the LGA, however, derives nonphysical terms in momentum conservation equations. It is the Lattice Boltzmann Method (LBM) that overcomes the drawback of the LGA. By replacing the particle occupation variables by a truncated power series of MaxwellBoltzmann distribution function, the LBM succeeded to completely eliminate the nonphysical terms of the $\mathrm{LGA}^{(3)}$. In the last several years, the LBM has succeeded in the application of multiphase fluid flows ${ }^{(4)}{ }^{-(10)}$. The LB models for isothermal two-phase flows and for ther- 
modynamics of ideal gas ${ }^{(11),(12)}$ have been independently proposed and successfully applied to several simulations. Although it was difficult to combine energy conservation with a twophase flow system, we presented a LB model for thermodynamics of two-phase flows ${ }^{(7)-(9)}$. The resulting macroscopic dynamics via the Chapman-Enskog expansion recover the full set of thermohydrodynamic equations for nonideal fluids. Numerical verification of fundamental properties of thermal fluids, including viscosity, thermal conductivity, and surface tension, agrees well with theoretical predictions. Direct numerical simulations of two-phase phenomena, including phase-transition, bubble deformation and droplet falling and bubble rising under gravity are carried out, demonstrating the applicability of the model. It was able to properly derive the pressure tensor based on van der Waals-Cahn-Hilliard free energy theory. By adopting FDLBM (Finite Difference Lattice Boltzmann Method) ${ }^{(13)}$ that interprets the LBM as a specific finite difference to a discrete-velocity Boltzmann equation, we enhanced the numerical stability, relaxed the restriction to relaxation time, and improved the accuracy. In this paper, we simulate a pool boiling with the LBM to verify the ability to simulate two-phase flows with phase transition.

Section II presents detailed description of the proposed LB model. Section III indicates numerical simulations, including a bubble rising and a pool boiling to demonstrate the applicability of this model for two-phase flows. It also shows that numerical measurement of thermal conductivity agrees well with theoretical prediction, and that surface tension calculated from this model follows Laplace's law.

\section{Lattice Boltzmann Method}

We will show a LBM for simulation of the following continuity equation:

$$
\frac{\partial \rho}{\partial t}+\frac{\partial \rho u_{\alpha}}{\partial x_{\alpha}}=0,
$$

the momentum conservation equation:

$$
\frac{\partial \rho u_{\alpha}}{\partial t}+\frac{\partial \rho u_{\alpha} u_{\beta}}{\partial x_{\beta}}=-\frac{\partial P_{\alpha \beta}}{\partial x_{\beta}}+\frac{\partial}{\partial x_{\alpha}}\left[\lambda \frac{\partial u_{\gamma}}{\partial x_{\gamma}}\right]+\frac{\partial}{\partial x_{\beta}}\left[\mu\left[\frac{\partial u_{\beta}}{\partial x_{\alpha}}+\frac{\partial u_{\alpha}}{\partial x_{\beta}}\right]\right],
$$

and the energy conservation equation:

$$
\frac{\partial \rho \varepsilon}{\partial t}+\frac{\partial \rho \varepsilon u_{\beta}}{\partial x_{\beta}}=-P_{\alpha \beta} \frac{\partial u_{\beta}}{\partial x_{\alpha}}+\frac{\partial}{\partial x_{\alpha}}\left[\chi \frac{\partial T}{\partial x_{\alpha}}\right]+\mu\left[\frac{\partial u_{\beta}}{\partial x_{\alpha}}+\frac{\partial u_{\alpha}}{\partial x_{\beta}}\right] \frac{\partial u_{\beta}}{\partial x_{\alpha}}+\lambda\left[\frac{\partial u_{\gamma}}{\partial x_{\gamma}}\right]^{2},
$$

for the two-phase flows based on van der Waals-Cahn-Hilliard free energy theory, where $\rho$ is the density, $u_{\alpha}$ is the local velocity, $\varepsilon$ is the internal energy, $P_{\alpha \beta}$ is the pressure tensor for the non-ideal flows, $\mu, \lambda$, and $\chi$ are the shear viscosity, the second viscosity, and the thermal diffusivity. $T$ is the nondimensional temperature, that is, $T=\hat{T} / T_{r}$. Here $\hat{T}$ and $T_{r}$ are the dimensional and the representative temperatures, respectively ${ }^{(14),(15)}$. The Greek subscripts, $\alpha, \beta$, and $\gamma$ denote the space directions in Cartesian coordinates. The pressure tensor in a non-uniform fluid $P_{\alpha \beta}$ is derived from the van der Waals-Cahn-Hilliard free energy ${ }^{(4)}$.

$$
P_{\alpha \beta}=\left[p-\kappa \rho \nabla^{2} \rho-\frac{\kappa}{2}|\nabla \rho|^{2}\right] \delta_{\alpha \beta}+\kappa\left(\frac{\partial \rho}{\partial x_{\alpha}}\right)\left(\frac{\partial \rho}{\partial x_{\beta}}\right),
$$

where $\kappa$ is the surface tension parameter that controls the strength of the surface tension, and $\delta_{\alpha \beta}$ is the Kronecker delta function, and the pressure, $p$, is

$$
p=\frac{\rho T}{1-b \rho}-a \rho^{2}
$$

given by the van der Waals equation of state as function of temperature, $T$, and density, $\rho$. The formulization of Eqs.(1)-(5) simulate phase transition or surface tension phenomena ${ }^{(4),(16),(17)}$. Here, $a \rho^{2}$ represents the effect of long-range attractive molecular forces, $b$ represents the influence of short-range repulsive forces. Although in general the van der Waals equation of state is not able to give quantitative features of real liquids, this equation of state is a 

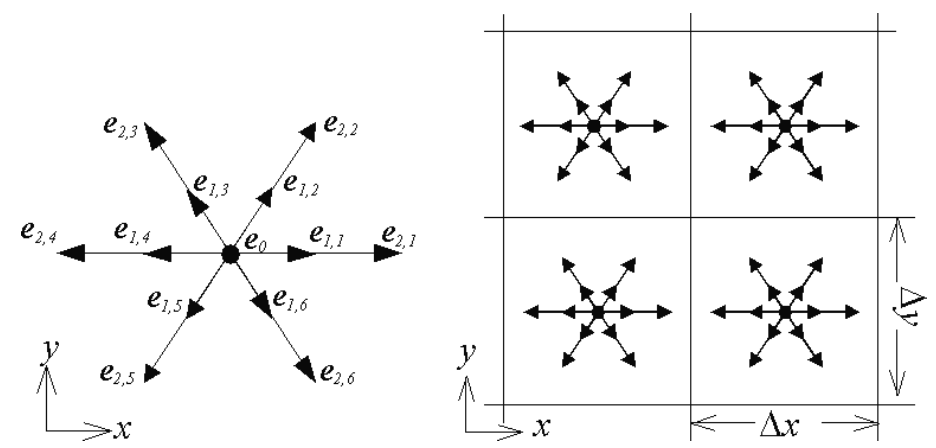

Fig. 1 Schematic of hexagonal lattices models.

reasonable model for both liquid and vapor properties. It gives relatively simple explanations for phenomena in real fluids, such as phase change and behavior near the critical point ${ }^{(4)}$.

The LBM resolved the problems of the lattice gas method: it removed statistical noise and eliminated the spurious invariants in the Navier-Stokes equations. The LB method could, however, be numerically unstable for high Reynolds number flows or for thermal problems. The numerical instability in the simulation of two-D and three-D flows has been studied ${ }^{(18)}$. Cao et al. proposed the FDLBM (Finite Difference Lattice Boltzmann Method) separating lattice symmetry from physical symmetry, and solved the numerical instability problem ${ }^{(13)}$. Lattice symmetry and Lagrangian nature of the scheme, which is used in the LBM, are directly associated with the property of particle dynamics. On the other hand, the physical symmetry is necessary to obtain the correct form of macroscopic momentum conservation equations from the kinetic equations of the LBM. As shown in Fig.1, in the FDLBM the physical symmetry is separated from the lattice symmetry, so that the grid size is able to be handled without relation to the structure of the discrete particle velocity. Regardless of the magnitude of triangle lattice (i.e., the magnitude of the discrete particle velocity), we can arbitrarily set the grid sizes, $\Delta x$ and $\Delta y$ (see Fig.1).

Instead of standard kinetic equation in the LBM, the FDLBM calculates the following approximated Boltzmann equation:

$$
\frac{\partial f_{\sigma, i}}{\partial t}+\mathbf{e}_{\sigma, i} \cdot \nabla f_{\sigma, i}=\Omega_{\sigma, i},(i=1,2, \cdots, N),
$$

where $f_{\sigma, i}$ is particle velocity distribution function, $\mathbf{e}_{\sigma, i}$ is the unit velocity vector along the $i$-th direction in space, $i$ is the velocity suffix, and $\Omega_{\sigma, i}$ is the collision operator. The FDLBM simulates fluid dynamics by solving the approximated Boltzmann equation (6) for particle velocity distribution function, $f_{\sigma, i}$, on the discrete lattice. Commonly the BGK approximation collision operator ${ }^{(3)}$,

$$
\Omega_{\sigma, i}=-\frac{f_{\sigma, i}-f_{\sigma, i}^{(0)}}{\tau \delta}
$$

is applied to the LBM. Here, $\tau$ is the relaxation time, $f_{\sigma, i}^{(0)}$ is the local equilibrium particle distribution function, and $\delta$ is a small parameter proportional to Knudsen number. Here, Eqs.(6) and (7) are nondimensional. The dimensional Boltzmann equation is written as

$$
\frac{\partial \hat{f}_{\sigma, i}}{\partial \hat{t}}+\hat{\mathbf{e}}_{\sigma, i} \cdot \hat{\nabla} \hat{f}_{\sigma, i}=-\frac{\hat{f}_{\sigma, i}-\hat{f}_{\sigma, i}^{(0)}}{\hat{\tau}}
$$

Substitution of $\mathbf{e}_{\sigma, i}=\hat{\mathbf{e}}_{\sigma, i} / e_{\sigma, r}, \nabla=L_{r} \hat{\nabla}, t=\hat{t} e_{\sigma, r} / L_{r}, \tau=\hat{\tau} / t_{r}$, and $f_{\sigma, i}=\hat{f}_{\sigma, i} / \rho_{r}$ into Eq.(8) gives Eqs.(6) and (7). Here $e_{\sigma, r}, L_{r}, t_{r}$, and $\rho_{r}$ are the representative discrete velocity, the representative length, the representative time, and the representative density, respectively. When we derive nondimensional equation (6) from Eq.(8), we use the relation $\delta=t_{r} e_{\sigma, r} / L_{r}$ that means the ratio of the mean freepath to the representative length. It is shown that $\delta$ corresponds to the Knudsen number. 
Starting from the LBGK equation, we construct a two-phase flow model that takes a consideration of the surface tension by adding a forcing term, $F_{\sigma, i}$, to the BGK collision operator. The evolution equation for two-phase thermohydraulics is written as

$$
\frac{\partial f_{\sigma, i}}{\partial t}+\mathbf{e}_{\sigma, i} \cdot \nabla f_{\sigma, i}=-\frac{f_{\sigma, i}-f_{\sigma, i}^{(0)}}{\tau \delta}+F_{\sigma, i} .
$$

Thermohydrodynamical quantities of density, $\rho$, momentum, $\rho u_{\alpha}$, and internal energy, $\varepsilon$ are determined through the moment of the equilibrium particle distribution, as

$$
\sum_{\sigma, i} f_{\sigma, i}=\rho, \quad \sum_{\sigma, i} f_{\sigma, i}\left(e_{\sigma, i}\right)_{\alpha}=\rho u_{\alpha}, \quad \frac{1}{2} \sum_{\sigma, i} f_{\sigma, i}\left|e_{\sigma, i}-u\right|^{2}=\rho \varepsilon .
$$

In order to simulate the isothermal two-phase flows based on the van der Waals-CahnHilliard free energy theory, Swift introduces the pressure tensor into the distribution function that satisfies the condition, $\Sigma_{\sigma, i}\left(e_{\sigma, i}\right)_{\alpha}\left(e_{\sigma, i}\right)_{\beta} f_{\sigma, i}=P_{\alpha \beta}+\rho u_{\alpha} u_{\beta}{ }^{(4)}$. This condition gives $\Sigma_{\sigma, i} f_{\sigma, i}\left|e_{\sigma, i}-u\right|^{2} / 2=\left(P_{x x}+P_{y y}\right) / 2$ that is inconsistent with Eq.(10). It is impossible to construct the thermal LBM for two phase flow by introduction of the surface tension effects into the distribution function as Swift did. From the above-mentioned reason, we use the strategy using the forcing term ${ }^{(5)}$ to propose the single component thermal LBM for two-phase flows.

This LB model uses a two-dimensional hexagonal lattice with 13 speeds: two kinds of moving particles with velocity vectors: $\mathbf{e}_{\sigma, i}=\sigma(\cos (\pi(i-1) / 3), \sin (\pi(i-1) / 3)),(i=$ $1, \cdots, 6),(\sigma=1,2)$, and one rest particle, $\left(e_{0}\right)_{\alpha}=0$, (see Fig.1). $\mathbf{e}_{\sigma, i} \sim{\sqrt{R T_{0}}}^{(14),(15)}$. Here, $\sigma$ is used as the magnitude of particle velocity, and $R$ is the gas constant.

The particle velocity equilibrium distribution function, $f_{\sigma, i}^{(0)}$, is given by a truncated power series of Maxwell-Boltzmann distribution in the local velocity $u$ assuming $|u| \ll 1$. The equilibrium distribution function for the rest particle is

$$
f_{0}^{(0)}=\rho\left(A_{0}+D_{0} u^{2}\right)
$$

and for the moving particle is given by

$$
\begin{aligned}
& f_{\sigma, i}^{(0)}=\rho\left(A_{\sigma}+B_{\sigma} \mathbf{e}_{\sigma, i} \cdot \mathbf{u}+C_{\sigma}\left(\mathbf{e}_{\sigma, i} \cdot \mathbf{u}\right)^{2}+D_{\sigma} u^{2}\right. \\
& \left.+E_{\sigma}\left(\mathbf{e}_{\sigma, i} \cdot \mathbf{u}\right)^{3}+F_{\sigma}\left(\mathbf{e}_{\sigma, i} \cdot \mathbf{u}\right) \mathbf{u}^{2}\right),
\end{aligned}
$$

and the coefficients are,

$$
\begin{aligned}
& A_{0}=1-\frac{5 \varepsilon}{2}+2 \varepsilon^{2}, A_{1}=\frac{4 \varepsilon(1-\varepsilon)}{9}, A_{2}=\frac{\varepsilon(4 \varepsilon-1)}{36}, B_{1}=\frac{4(1-\varepsilon)}{9}, \\
& B_{2}=\frac{(4 \varepsilon-1)}{36}, C_{1}=\frac{4(2-3 \varepsilon)}{9}, C_{2}=\frac{6 \varepsilon-1}{72}, D_{0}=-\frac{5}{4}+2 \varepsilon, \\
& D_{1}=-\frac{2(1-\varepsilon)}{9}, D_{2}=-\frac{(4 \varepsilon-1)}{72}, E_{1}=-\frac{4}{27}, E_{2}=\frac{1}{108}, F_{1}=0, F_{2}=0 .
\end{aligned}
$$

The second viscosity, the shear viscosity, and the diffusivity are given by $\lambda=-\rho \varepsilon \tau$, $\mu=\rho \varepsilon \tau$, and $\chi=2 \rho \varepsilon \tau$ through the Chapmann-Enskog procedure ${ }^{(9)}$.

Comparing with Eqs.(1)-(3) and kinetic equations derived from Eq.(9), one gets constraints for the $F_{\sigma, i}$ as

$$
\begin{gathered}
\sum_{\sigma, i} F_{\sigma, i}\left(\begin{array}{c}
1 \\
\left(e_{\sigma, i}\right)_{\alpha} \\
\left|e_{\sigma, i}\right|^{2} / 2 \\
\left|e_{\sigma, i}\right|^{2}\left(e_{\sigma, i}\right)_{\alpha}
\end{array}\right)=\left(\begin{array}{c}
a_{1} \\
\left(a_{2}\right)_{\alpha} \\
a_{3} \\
\left(a_{4}\right)_{\alpha}
\end{array}\right) . \\
\left(\begin{array}{c}
a_{1} \\
\left(a_{2}\right)_{\alpha} \\
a_{3} \\
\left(a_{4}\right)_{\alpha}
\end{array}\right)=\left(\begin{array}{c}
0 \\
\partial_{\alpha} \rho \varepsilon-\partial_{\beta} P_{\alpha \beta} \\
\partial_{\alpha} \rho \varepsilon u_{\alpha}-\partial_{\alpha} P_{\beta \alpha} u_{\beta} \\
4 \varepsilon\left(\partial_{\beta} \rho T \delta_{\alpha \beta}-\partial_{\beta} P_{\alpha \beta}\right)
\end{array}\right) .
\end{gathered}
$$




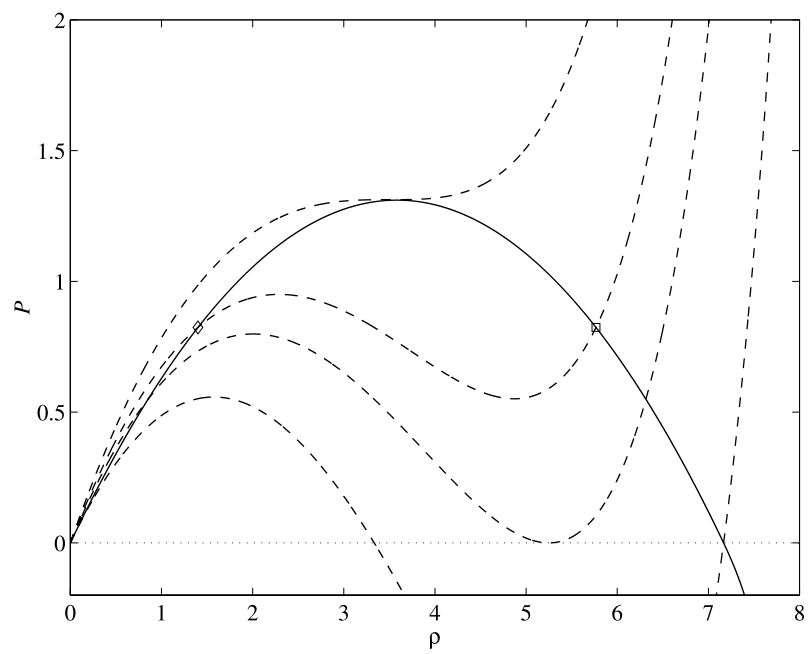

Fig. 2 Isotherms (dashed lines) for the van der Waals equation of state for various temperatures in $P-\rho$ coordinates. The densities of coexisting points at temperature $T=0.9$ are $1.400(\diamond)$ and 5.767(ם), respectively.

The coefficients, $a_{1}-a_{4}$, correspond to a difference between the equations for ideal gas and Eq.(1)-(3) for the two-phase flows. For instants, the constraints, $\left(a_{2}\right)_{\alpha}=\partial_{\alpha} \rho \varepsilon-\partial_{\beta} P_{\alpha \beta}$, in Eq.(15) is derived from the difference of the momentum equations between for the ideal gas and for van der Waals fluids.

Inclusion of the first and the second rank tensors as

$$
F_{\sigma, i}=\left(G_{\sigma}\right)_{\alpha}\left(e_{\sigma, i}\right)_{\alpha}+H_{\sigma}\left|e_{\sigma, i}\right|^{2},
$$

enables the fictitious forcing term to satisfy the constraints, Eq.(15). Due to the first and second rank tensors, $F_{\sigma, i}$ can contribute to the derivation of the mass, momentum and energy equations. In each derivations, the effects of $F_{\sigma, i}$ are suitably controlled by the coefficients, $G_{\sigma}, H_{\sigma}$ depending on the magnitude of velocity, $\sigma$. For instance, in the derivation of the mass equation, $H_{\sigma}$ can adjust the effect of the second term in Eq.(16) to be zero. Likewise, the first term with $G_{\sigma}$ in Eq.(16) is formalized to set to recover correct form not only of the pressure tensor in Eq.(2) but also of the diffusivity in Eq.(3) at the macroscopic level. The second term in Eq.(16) can give the appropriate pressure tensor in the energy equation. Inserting Eq.(16) to Eq.(13), one obtains the coefficients, $G_{\sigma}, H_{\sigma}$ as follows:

$$
\left(\begin{array}{c}
\left(G_{1}\right)_{\alpha} \\
\left(G_{2}\right)_{\alpha} \\
H_{1} \\
H_{2}
\end{array}\right)=\left(\begin{array}{c}
\left(4\left(a_{2}\right)_{\alpha}-\left(a_{4}\right)_{\alpha}\right) / 9 \\
\left(\left(a_{4}\right)_{\alpha}-\left(a_{2}\right)_{\alpha}\right) / 36 \\
\left(2 a_{1}-a_{3}\right) / 9 \\
\left(2 a_{3}-a_{1}\right) / 72
\end{array}\right) .
$$

This formulation actualizes the LB model capable of simulating thermohydrodynamic two-phase flows shown in Eq.(1)-(3).

\section{Numerical Experiments}

Discretization is necessary to calculate the evolution equation (9). The convection term in Eq.(9) is discretized by the second order centered difference for the simulation of Couette flow. For steep density gradients problem, e.g. interfaces in two-phase flows, the second order centered difference generates numerical oscillation. Applying a first order upwind difference to Eq.(9) leads to the stable simulation for two-phase flows without any numerical oscillations (7). For the following simulations of two-phase flows, we will utilize the first order upwind difference for the discretization of the convection term. The Pressure tensor shown in Eq.(4) is also discretized by the second order centered difference scheme. The temporal discretization 


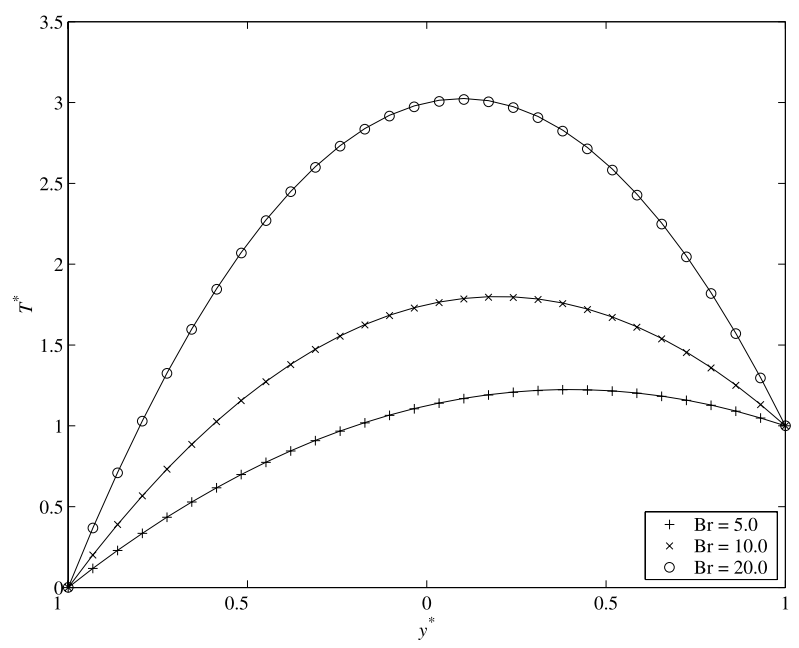

(a) The density $\rho$ equals to 1.400 .

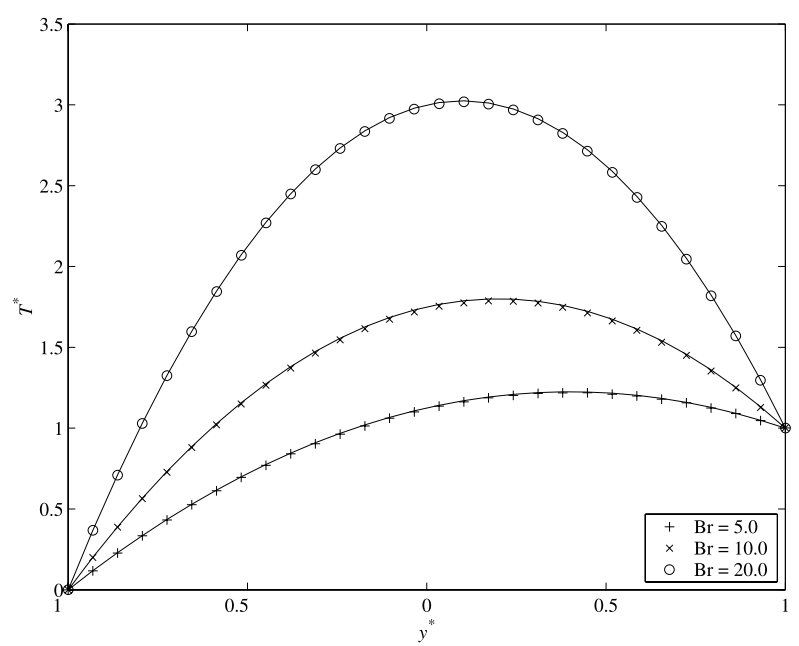

(b) The density $\rho$ equals to 5.767 .

Fig. 3 Simulation of Couette flow.

is obtained using the second order Runge-Kutta (or the modified Euler) method. The time evolution of particle distribution functions is derived using

$$
\begin{aligned}
& f_{\sigma, i}^{n+1 / 2}=f_{\sigma, i}^{n}+\frac{1}{2} \Delta t\left(-\mathbf{e}_{\sigma, i} \cdot \nabla f_{\sigma, i}^{n}-\frac{f_{\sigma, i}^{n}-f_{\sigma, i}^{(0), n}}{\tau \delta}+F_{\sigma, i}^{n}+F_{g}^{n}\right), \\
& f_{\sigma, i}^{n+1}=f_{\sigma, i}^{n}+\Delta t\left(-\mathbf{e}_{\sigma, i} \cdot \nabla f_{\sigma, i}^{n+1 / 2}-\frac{f_{\sigma, i}^{n+1 / 2}-f_{\sigma, i}^{(0), n+1 / 2}}{\tau \delta}+F_{\sigma, i}^{n+1 / 2}+F_{g}^{n+1 / 2}\right) .
\end{aligned}
$$

We summarize the algorithm of computation.

Step 1. Set $f_{\sigma, i}^{(0), 1}$ corresponding to the initial condition, $\rho^{1}, u_{\alpha}^{1}$, and $\varepsilon^{1}$. The initial values for the distribution function are given by $f_{\sigma, i}^{1}=f_{\sigma, i}^{(0), 1}$.

Step 2. Equations (18) and (19) compute $f_{\sigma, i}^{n+1}$, and Eq.(10) gives $\rho^{n+1}, u_{\alpha}^{n+1}$, and $\varepsilon^{n+1}$.

Step 3. For two-phase flows simulations, the boundary values for the distribution function are given by $f_{\sigma, i}^{n+1}=f_{\sigma, i}^{(0), n+1}$. For single phase flow, the extrapolation scheme is used ${ }^{(19)}$.

Step 4. Compute $f_{\sigma, i}^{(0), n+1}$ and $F_{\sigma, i}^{n+1}$ by Eqs.(11), (12), and (16).

Step 5. Advance one time step and return to step 2.

To verify the thermal properties of the model, we simulate the Couette flow for fluids in the liquid state and the vapor state. Each state can be determined by Maxwell construction 


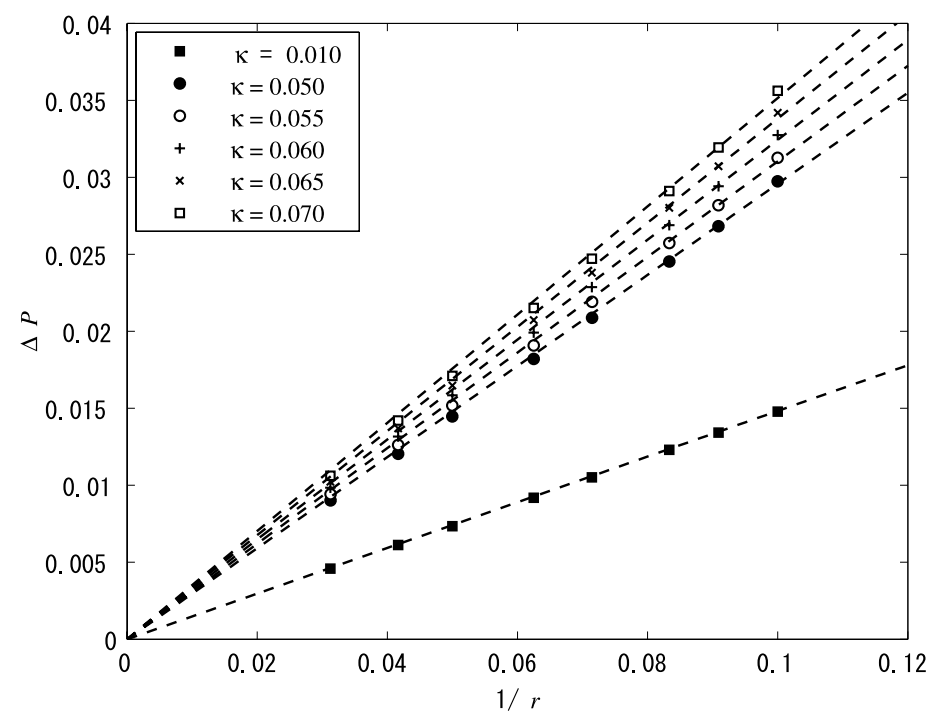

Fig. $4 \Delta \mathrm{P}$ vs $1 / r$

for the van der Waals equation of state with the parameters $a=9 / 28, b=2 / 21$ (see Fig.2). In Fig.2, the solid line is the coexistence curve numerically determined by Maxwell's equal area rule ${ }^{(4)}$. We set the starting temperature 0.9 , which is close to the critical temperature, $T_{c}=1.0$, because the van der Waals equation of state gives reasonable results near the critical point. The starting liquid density $\rho_{G}$ is 5.767 ( $\diamond$ in Fig.2) and vapor density $\rho_{L}$ is 1.400( $\square$ in Fig.2) for a given temperature, $T=0.9$. The following simulations will use these values for $a, b, \rho_{G}, \rho_{L}$, and $T$.

With a small temperature gradient, the normalized temperature distribution has an analytical solution written as

$$
T^{*}=\frac{T-T_{0}}{T_{1}-T_{0}}=\frac{1}{2}\left(1+y^{*}\right)+\frac{\mathrm{Br}}{8}\left(1-y^{* 2}\right),
$$

where $y^{*}$ is the normalized distance from the center of the channel, and $\mathrm{Br}$ is Brinkman number, the product of the Prandtl and Eckert numbers. $T_{1}$ and $T_{0}$ are the temperatures of the top and bottom walls, respectively. The second-order extrapolation scheme for boundary condition proposed by $\mathrm{S}$. Chen is used for the walls ${ }^{(19)}$. The simulations are carried out for the liquid and vapor phases. A very good agreement is seen in Fig.3 (a) and (b) between the analytical and numerical solutions for both densities. It could be concluded from the simulation results of the Couette flows that the proposed LB model is valid in simulating flows with thermal diffusion.

The next numerical experiment is concerned with Laplace's law to verify the surface tension on an interface in two-phase flows. Far from the interface, the fluid obeys the usual Navier-Stokes equation, however, nearby the interface, the interface behavior is important, and the surface tension should be appropriately calculated by the model. The mechanical definition of surface tension $\sigma_{s}$ that is, Laplace's law is

$$
\Delta P=\frac{\sigma_{s}}{r},
$$

where $r$ is dimensionless radius of the spherical bubble, and $P$ is the dimensionless pressure difference between the inside and outside of a single-component bubble. Equation (21) indicates that pressure difference, $\Delta P$, is proportional to the curvature of droplet, $1 / r$. Initially one bubble with radius, $r$, is surrounded by the vapor. The grid points are $(4 r / \Delta x) \times(4 r / \Delta y)$ so that one side of simulation domain is twice as long as diameter of the droplet. Figure 4 shows the relation between curvature $1 / r$ and pressure difference $\Delta P$ for a wide rage of the surface tension parameter, $\kappa$. The results are obtained from the numerical experiments for 


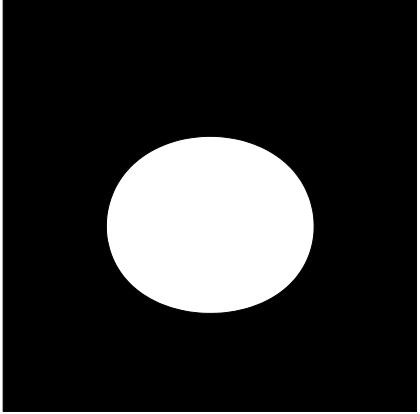

(a) $\mathrm{Eo}=0.808, \mathrm{M}=4.33 \times 10-5, \mathrm{Re}=29.28$

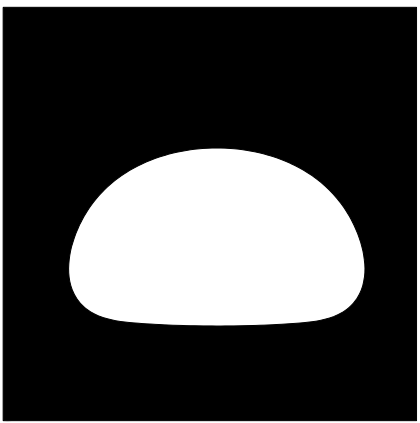

(c) $\mathrm{Eo}=3.089, \mathrm{M}=2.88 \times 10-6, \mathrm{Re}=91.10$

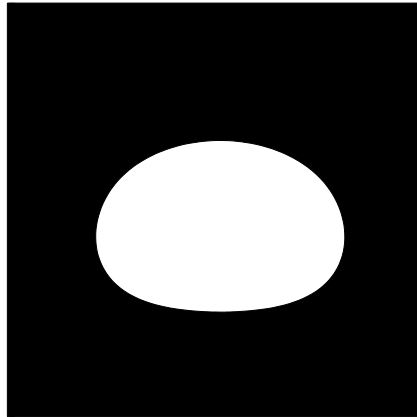

(b) $\mathrm{Eo}=1.589, \mathrm{M}=8.85 \times 10-5, \mathrm{Re}=22.65$

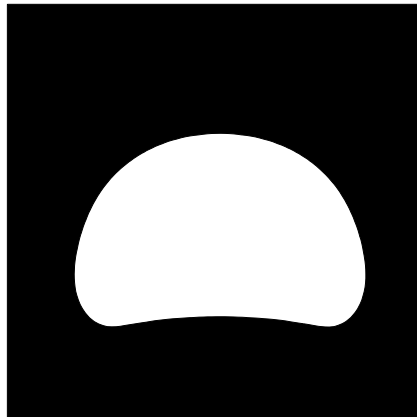

(d) $\mathrm{Eo}=7.339, \mathrm{M}=1.92 \times 10-3, \mathrm{Re}=114.84$

Fig. 5 The density profiles of a rising bubble.

bubbles with various radii. As is evident from Fig.4, this LBM reproduces surface tension phenomenon which is significant for elucidating two-phase fluid.

We simulate a bubble rising in viscous liquid to verify a dependence of shapes of the bubble on terminal velocities. As the initial condition, the diameter of a bubble is set to $48 \times \Delta x$, so that it is half of the width of simulation domain where grid size is $96 \times 96 \times \Delta x^{2}$. One bubble located in the liquid rises due to buoyancy. Etövos number Eo, Morton number $\mathrm{M}$, Reynolds number Re , are given by

$$
\text { Eo }=\frac{g\left(\rho_{L}-\rho_{G}\right) D^{2}}{\sigma_{s}}, \quad \mathrm{M}=\frac{g \mu_{L}^{4}\left(\rho_{L}-\rho_{G}\right)}{\rho_{L}^{2} \sigma_{s}^{3}}, \quad \operatorname{Re}=\frac{\rho_{L} U_{t} D}{\mu_{L}},
$$

where $g$ is the acceleration gravity, $D$ is the volume equivalent diameter of a bubble, and $U_{t}$ is the terminal rising velocity. Eo is ratio of a Weber to a Froude number; it is a measure of the relative magnitude of gravity and interfacial tension forces, and $\mathrm{M}$ is effects of propriety of each phase ${ }^{(20)}$. Grace, Bhaga and Weber studied a general graphical correlation to predict the shape and terminal rising velocity of any bubble in terms of the dimensionless groups, $\mathrm{M}$, Eo, and $\mathrm{Re}^{(20),(21)}$. For the LBM, the gravity accelerator is given by adding

$$
F_{g}=\frac{\left(e_{\sigma, i}\right)_{\alpha} g_{\alpha}}{15}\left(\rho_{L}-\rho_{G}\right)
$$

to Eq.(9) ${ }^{(22)}$. The surface tension which is necessary to calculate the Eo and M is calculated by Laplace's law, Eq.(21). Figure 5 shows the density distributions of the simulation with the high density in black and low density in white. Here, $\kappa=0.050, \tau=0.16, \Delta t=0.1$, $\Delta x=\Delta y=0.5, g=0.0003$. It reveals that while Etövos number increases, the bubble deforms from the spherical to ellipsoidal shape, and finally it becomes oblate ellipsoidal cap. The simulation results are almost equal to experimental results shown in the shape regime map in Ref. (21).

To substantiate the applicability of this LB model to simulation of boiling, we conduct the numerical experiments of bubble generation near a hot wall. Initially the calculation region is filled with only liquid and the temperature of one part of bottom wall is equal to 1.0, while the temperature of the other region of the wall is 0.9 . Figure 6 shows time evolution of the density 


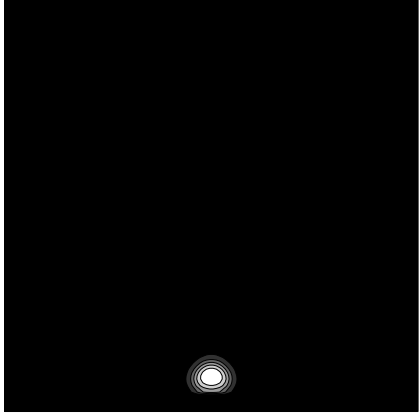

(a) $\mathrm{t}=50$

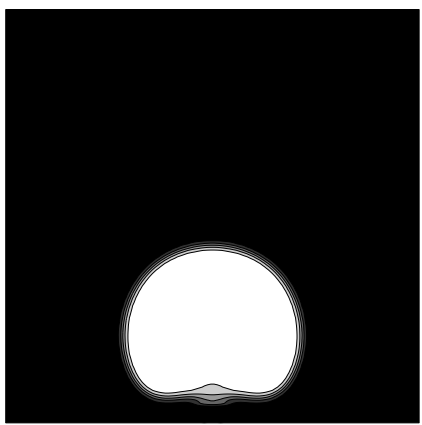

(c) $\mathrm{t}=300$

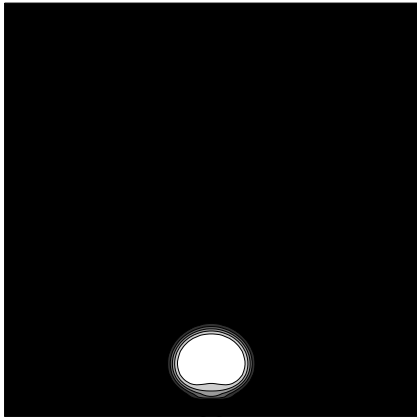

(b) $\mathrm{t}=100$

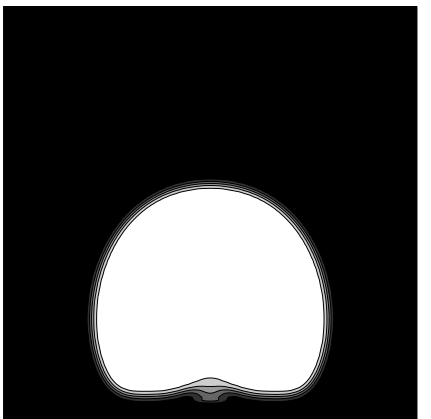

(d) $\mathrm{t}=500$

Fig. 6 Snapshots of density field of a bubble generation.

distribution of the simulation in gray scale as Fig.5. As shown in Fig.6, the heat flux from the hot part of the wall $(T=1.0)$ generates a bubble. At a high temperature, the attraction among molecules is weak and the random motion of molecules is dominant ${ }^{(23)}$. In this simulation, the hot wall with the critical temperature makes the fluid exist in a single phase. Once the temperature is quenched below the critical value by the cool liquid $(T=0.9)$, the fluid enters the interface region of the van der Waals fluid that is mechanically unstable $(\partial P / \partial \rho>0)$, as shown in Fig.2. This unstable state keeps the phases separated, mimimizes the free energy, and warrants the sharp interface. The enthalpy is defined by

$$
h=\varepsilon+P / \rho,
$$

and the difference between the enthalpy of two phases determines latent heat, $L$,

$$
L=h_{G}-h_{L}
$$

Figure 7 (a) and (b) show the density and enthalpy distribution of the bubble at the time $t=500$ in the bubble generation simulation, respectively. Comparing Fig.7(a) with (b), one can observe the difference of the enthalpy between the gas and the liquid phases, and also confirm the generation of the latent heat corresponding to Eq.(25). The LB model has capability to simulate the latent heat by introducing pressure tensor based on van der Waals-Cahn-Hilliard free energy theory.

To substantiate the applicability of this LBM for the two-phase flows simulation, we calculate a bubble generation under gravity. Initially there is only stationary liquid in a simulation domain whose grid size is $64 \times 128$. The stationary boundary $(T=0.9)$ condition and the periodic boundary condition are applied in the $x$ direction and the y direction, respectively. At the position $(x=32)$ on the bottom wall, the temperature gradient is set to be constant to make a heat flux constant. The density, temperature, and velocity distributions at different time steps are indicated in the Fig.8, Fig.9, and Fig.10, respectively. In Fig.8 and 9, the variation of the density and temperature is shown in gray scale with the minimum in white and the maximum in black. Figure 8 (a) and (b) show that the bubble generates in the vicinity of the hot part of the bottom wall. When the bubble grows to the size that the buoyancy is 


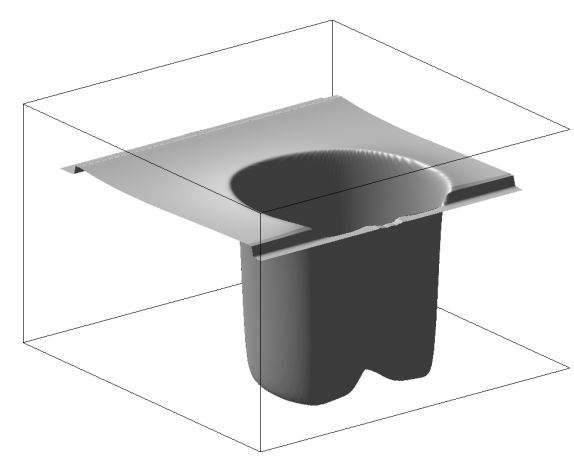

(a) Density

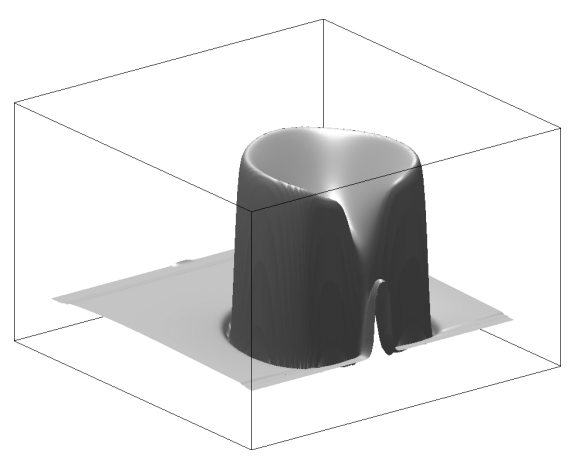

(b) Enthalpy

Fig. 7 The density and enthalpy distribution of the bubble generation at time, $\mathrm{t}=500$.

greater than the surface tension, it began to departure from the wall (Fig.8 (d) and (e)). In Fig. 8 (d), the slow growth speed of the top of the bubble and bubble generation in vicinity of the wall produce a coned bottom of the bubble. As shown in Fig.8 (e) and (f), when the bubble departures from the bottom wall, a coned bottom of the bubble rapidly shrinks. After the departure of the first bubble, there is the dormant period before a generation of the next bubble. Figure 9 (f) and (g) show that the thermal boundary layer is raised by the shrink of the bottom of the bubble at the departure. After the departure of the bubble, the cool liquid flows under the bubble, and the thermal boundary layer becomes flat (see Fig.9 (h)). Figure 10 (a) shows that the generating bubble excludes the liquid, and produces the flow streaming out of the bubble. When the bubble departures from the wall, circulating flows are produced around the bubble (see Fig.10 (b)). As indicated in Fig.10 (b) and (c), while the bubble departures from the wall, a symmetric wake occurs under the bubble. As is evident from Fig.10 (d), the first bubble disturbs the next generating bubble. The density, temperature, and velocity distributions calculated by the LB model are similar to the experiments results. The simulation results shown in Figs.6-10 demonstrate that Eqs.(6), (7) are able to calculate simultaneously mass and energy transportation including the surface tension effect. The introduction of the surface tension energy and of the van der Waals force induces the difference of temperature between liquid and vapor phases shown in Fig.9. It would be reasonably summarized that this LB model is applicable to the simulation of two-phase flows.

\section{Conclusions}

We indicated a LBM capable of simulating thermohydrodynamics for two-phase flows and pressure tensor derived from van der Waals-Cahn-Hlliard free energy theory. This model recovered the correct form of the macroscopic equations (1)-(3) for two-phase flows to the second order. The numerical experiments, including a bubble rising and a pool boiling, demonstrated the applicability of this model for two-phase flows. These numerical experiments revealed that this LB model autonomously simulated complex interface behavior without explicitly calculating experimental equations for interface phenomena or Laplace's law. The simulation results of the Couette flow also elucidated that the proposed model is valid in simulating flows with thermal diffusion.

The LBM was able to calculate the oblate ellipsoidal cap bubble for the $\operatorname{Re}=110$ in the simulation of bubble rising. To simulate a spherical cap bubble rising in the pool boiling phenomena, however, the Etövos number and Reynolds number should be more than 40 and more than 100, respectively. To numerically stably simulate such high numbers with this LB model, we need computer power to be able to use vast amounts of grid size or introduce turbulence models into the LBM.

In the simulations indicated above, the liquid density is 5.767 and vapor density is 1.400 , and the density ratio is approximately 4.0. That is not practical for simulation of liquid-gas two-phase flows at ordinary temperatures. To simulate the two-phase flows at room tempera- 


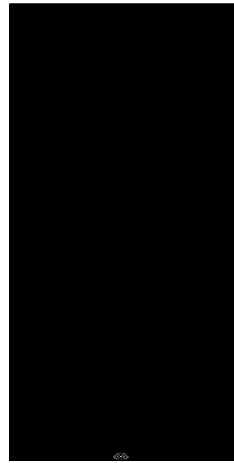

(a) $t=1$

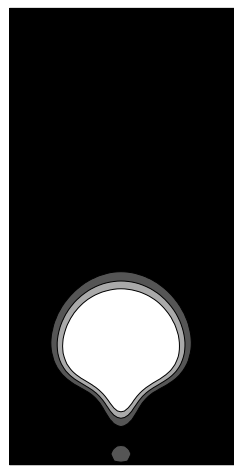

(e) $\mathrm{t}=2,100$

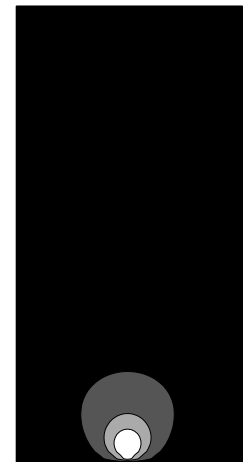

(b) $\mathrm{t}=1,000$

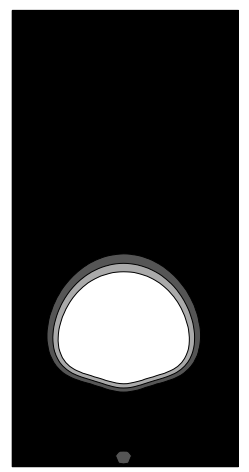

(f) $\mathrm{t}=2,125$

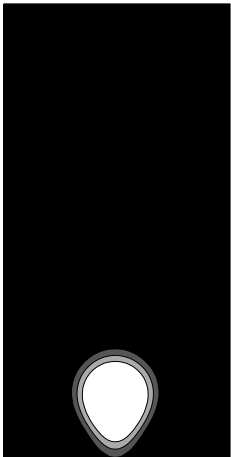

(c) $t=2,000$

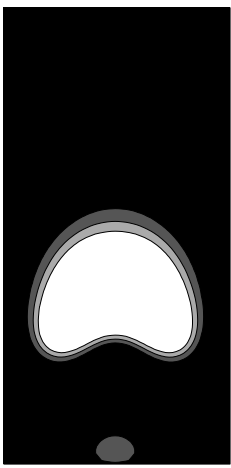

(g) $\mathrm{t}=2,175$

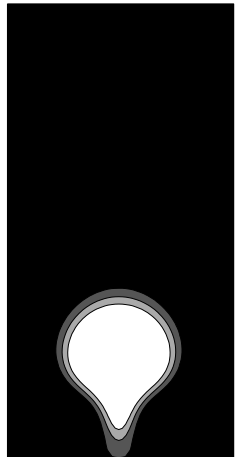

(d) $\mathrm{t}=2,075$

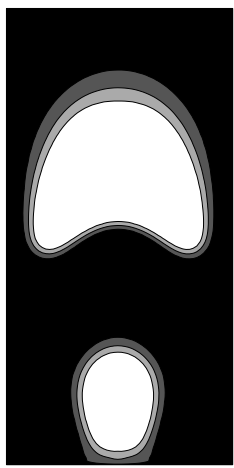

(h) $t=2,350$

Fig. 8 Snapshots of density field of the pool boiling.

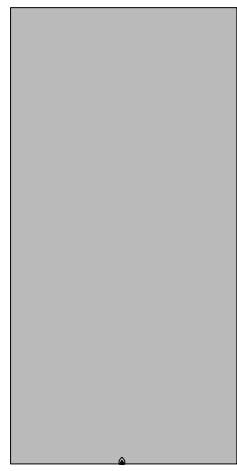

(a) $t=1$

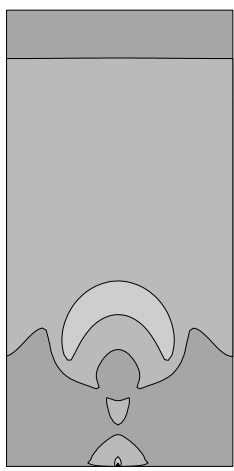

(e) $\mathrm{t}=2,100$

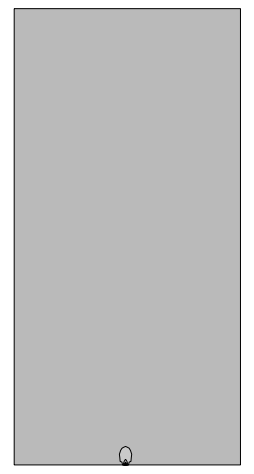

(b) $\mathrm{t}=1,000$

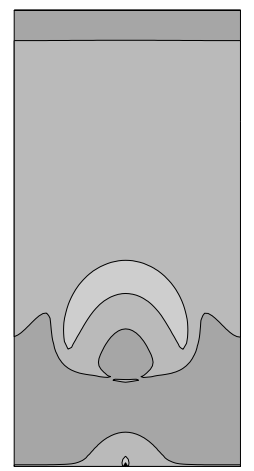

(f) $\mathrm{t}=2,125$

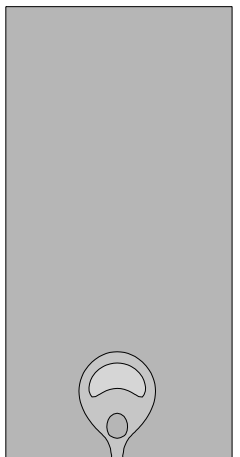

(c) $\mathrm{t}=2,000$

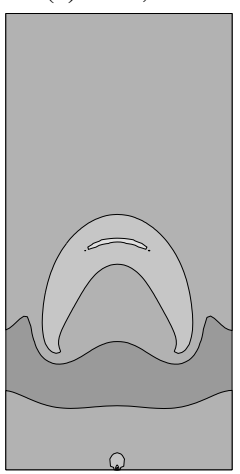

(g) $\mathrm{t}=2,175$

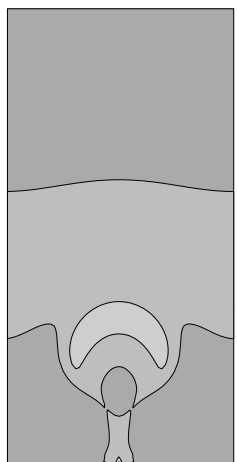

(d) $\mathrm{t}=2,075$

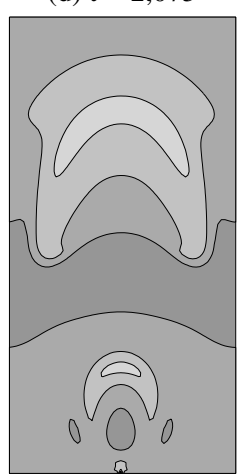

(h) $\mathrm{t}=2,350$

Fig. 9 Snapshots of temperature field of the pool boiling. 


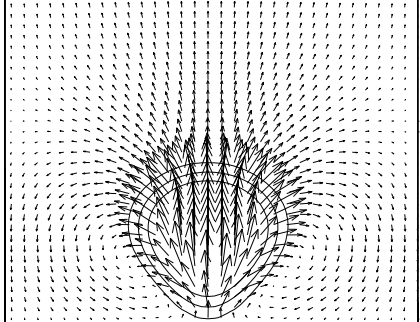

(a) $\mathrm{t}=2,000$

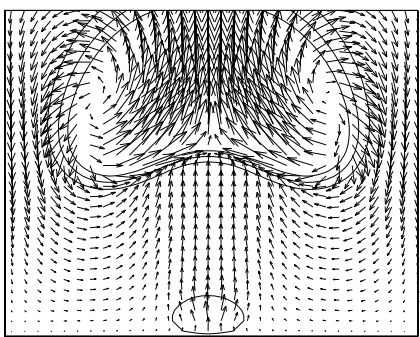

(c) $\mathrm{t}=2,175$

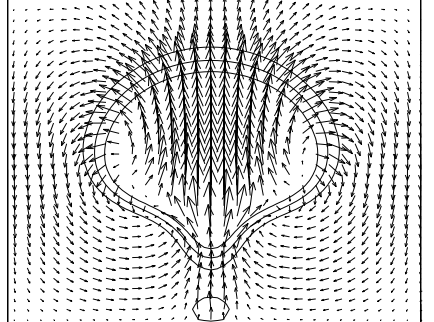

(b) $\mathrm{t}=2,100$

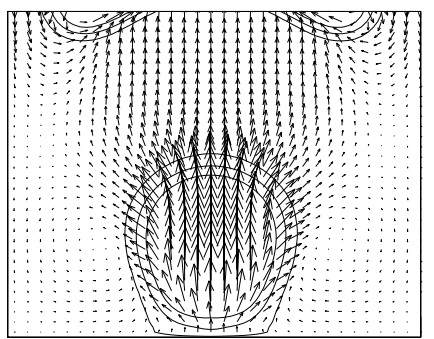

(d) $\mathrm{t}=2,350$

Fig. 10 Snapshots of velocity field of the pool boiling.

ture, the proposed LB model should stably calculate high density ratio. Although high density ratio between the vapor and the liquid is obtained by the decrease in temperature, the equation of state has minus pressure at the temperature less than 0.84375 as shown in Fig.2. To avoid the instabilities induced by the minus pressure, we used the temperature as 0.9. Recently Inamuro et al. has proposed lattice Boltzmann method capable of calculating high density ratio using additional distribution function for index function ${ }^{(24)}$.

\section{Nomenclature}

$D$ : volume equivalent diameter

Eo : Etövos number

$\mathbf{e}_{\sigma, i}:$ discrete velocity vector

$e_{\sigma, r}:$ representative discrete velocity

$f_{\sigma, i}:$ distribution function

$f_{\sigma, i}^{(0)}$ : local equilibrium distribution function

$F_{\sigma, i}:$ forcing term

$g:$ acceleration gravity

$h:$ enthalpy

$i$ : suffix for discrete velocity direction

$L$ : latent heat

$L_{r}$ : representative length

$\mathrm{M}$ : Morton number

$p:$ pressure given by the van der Waals equation of state

$P_{\alpha \beta}$ : pressure tensor derived from the van der Waals-Cahn-Hilliard free energy

Re : Reynolds number

$T$ : temperature

$T_{r}$ : representative temperature

$t_{r}$ : representative time

$u_{\alpha}:$ fluid velocity

$U_{t}:$ terminal rising velocity

$\chi:$ thermal diffusivity

$\varepsilon$ : internal energy

$\kappa:$ surface tension parameter

$\lambda$ : second viscosity

$\mu$ : shear viscosity

$\rho:$ fluid density

$\rho_{r}$ : representative density

$\sigma$ : suffix for discrete velocity magnitude

$\tau$ : relaxation time

$\Omega_{\sigma, i}:$ collision operator

\section{Acknowledgements}

We thank Prof. R. Takahashi and Prof. E. Takegoshi for thoughtful and helpful comments.

\section{References}

( 1 ) Matsukuma, Y. and Abe, Y., Lattice Gas Automata Simulations of Flow through Porous Media, Proc. of the Workshop on Severe Accident Research, (1998), pp.128-133. 
( 2 ) Niimura, H., Lattice-Gas Model with Wetness Control for Various Deformable Microstructures, Comp. Phys. Comm., Vol.129 (2000), pp.145-157.

( 3 ) McNamara, G. R. and Zanetii, G., Use of the Boltzmann Equation to Simulate LatticeGas Automata, Phys. Rev. Lett., Vol.61(1988), pp.2332-2335.

( 4 ) Swift, M. R., Osborn, W. R., and Yeomans, J. M., Lattice Boltzmann Simulation of Nonideal Fluids, Phys. Rev. Lett., Vol.75 (1995), pp.830-833.

( 5 ) Qian, Y. H. and Chen, S., Finite size effect in lattice BGK models, Int. J. Modern Phys. $C$, Vol.8 (1997), pp.763-772.

( 6 ) Seta, T., Kono, K., Martinez, D., and Chen, S., Lattice Boltzmann Scheme for Simulating Two-Phase Flows, JSME Int. J. B, Vol.43 (2000), pp.305-313.

( 7 ) Seta, T. and Kono, K., Thermal Lattice Boltzmann Method for Liquid-Gas Two-Phase Flows in Two Dimension, JSME Int. J. B, Vol. 47 No.3 (2004), pp.572-583.

( 8 ) Seta, T., Kono, K., and Chen S., Lattice Boltzmann Method for Two-phase Flows, Int. J. Modern Phys. B, Vol.17 (2003), pp.169-172.

( 9 ) Seta, T., Ryoichi, T., and Okui, K., Takegoshi, E.., Thermal Lattice Boltzmann Model for Simulating Two-Phase Flows (in Japanese), Trans. JSME B, Vol. 68 No.672 (2002), pp.2186-2194.

(10) Takada, N., Misawa, M., Tomiyama, A., and Fujiwara, S., Numerical Simulation of Two- and Three-Dimensional Two-Phase Fluid Motion by Lattice Boltzmann Method, Comp. Phys. Comm., Vol.129 (2000), pp.233-246.

(11) Alexander, F. J., Chen, S., and Sterling, J. D., Lattice Boltzmann Thermohydrodynamics, Phys. Rev. E, Vol.47 (1993), pp.2249-2252.

(12) Chen, Y., Ohashi, H., and Akiyama, M., Thermal Lattice Bhatnagar-Gross-Krook Model without Nonlinear Deviations in Macro-Dynamic Equations, Phys. Rev. E, Vol.50 (1994), pp.2776-2783.

(13) Cao, N., Chen, S., Jin, S., and Martinez, D., Physical Symmetry and Lattice Symmetry in the Lattice Boltzmann Method, Phys. Rev. E, Vol.55 (1997), pp.21-24.

(14) Onishi, J., Chen, Y., and Ohashi, H., Lattice Boltzmann simulation of natural convection in a square cavity, JSME Int. J. B, Vol. 44 No.1 (2001), pp.53-62.

(15) Kataoka, T. and Tsutahara, M., Lattice Boltzmann model for the compressible Navier-Stokes equations with flexible specific-heat ratio, Phys. Rev. E, Vol.69 (2004), pp.035701-1-035701-4.

(16) Takada, N. and Tomiyama, A., A Numerical Method for Two-Phase Flow Based on a Phase-Field Model, JSME Int. J. B, Vol.49 (2006), pp.636-644.

(17) Nadiga, B. T. and Zaleski, S., Investigations of a two-phase fluid model, Eur. J. Mech. B/Fluids, Vol.15 (1996), pp.885-896.

(18) Sterling, J. D. and Chen, S., Stability Analysis of Lattice Boltzmann Methods, J. Comput. Phys., Vol.123 (1996), pp.196-206.

(19) Chen, S., Martínez, D., and Mei, R., On boundary conditions in lattice Boltzmann methods, Phys. Fluids, Vol.8 No.9 (1996), pp.2527-2536.

(20) Grace, J. R., Shapes and Velocities of Bubbles Rising in Infinite Liquids, Trans. Instn. Chem. Engrs., Vol.51 (1973), pp.116-120.

(21) Bhaga, D. and Weber, M. E., Bubbles in Viscous Liquids:Shapes, Wakes and Velocities, J. Fluid Mech., Vol.105 (1981), pp.61-85.

(22) Buick, J. M. and Greated, C. A., Gravity in a lattice Boltzmann model, Phys. Rev. E, Vol.61 (2000), pp.5307-5320.

(23) He, X., Chen, S., and Zhang, R., A lattice Boltzmann scheme for incompressible multiphase flow and its application in simulation of Rayleigh-Taylor instability, J. Comput. Phys., Vol.152 (1999), pp.642-663.

(24) Inamuro, T., Ogata, T., Tajima, S., and Konishi, N., A lattice Boltzmann method for immiscible two-phase flows with large density differences, J. Comput. Phys., Vol.198 (2004), pp.628-644. 